\title{
THE
}

4-15-1985

\section{Heisenberg Spin Chains: Quantum-Classical Crossover and the Haldane Conjecture}

\author{
J. B. Parkinson \\ Jill C. Bonner \\ University of Rhode Island \\ Gerhard Müller \\ University of Rhode Island, gmuller@uri.edu \\ M. P. Nightingale \\ University of Rhode Island, nightingale@uri.edu \\ H. W.J. Blöte
}

Follow this and additional works at: https://digitalcommons.uri.edu/phys_facpubs

Terms of Use

All rights reserved under copyright.

\section{Citation/Publisher Attribution}

J. B. Parkinson, J. C. Bonner, Gerhard Müller, M. P. Nightingale and H. W. J. Blöte. Heisenberg spin chains: quantum classical crossover and the Haldane conjecture. J. Appl. Phys. 57 (1985), 3319-3321. Available at: http://dx.doi.org/10.1063/1.335134 


\title{
Heisenberg spin chains: Quantum-classical crossover and the Haldane conjecture
}

\author{
J. B. Parkinson ${ }^{a)}$ and J. C. Bonner ${ }^{\text {b) }}$ \\ Department of Physics and Astronomy, Michigan State University, East Lansing, Michigan 48824
}

G. Müller and M. P. Nightingale

Physics Department, University of Rhode Island, Kingston, Rhode Island 02881

H.W. J. Blöte

Technische Hogeschool Delft, Delft, Netherlands

\begin{abstract}
A comprehensive investigation has been made of the spectral excitations and static properties of Heisenberg antiferromagnetic chains of spin 1/2,1,3/2, and 2, using Lanczös, Bethe Ansatz, and Monte Carlo techniques. An unusual and unanticipated crossover mechanism for spin chains with $1 / 2 \leqslant S \leqslant \infty$ has been discovered. The validity of the Haldane conjecture concerning the presence of a spectral excitation gap for integer-spin chains has been investigated by exact finite chains calculations of (a) the primary singlet-triplet excitation gap, (b) higher excitation gaps, and (c) the Fourier transform of the ground state correlation functions. A new Monte Carlo method has extended the spin-1 gap calculations to $N=32$.
\end{abstract}

A considerable degree of interest has been generated recently by a claim of Haldane ${ }^{1}$ that integer-spin Heisenberg antiferromagnetic chains show qualitatively different phase behavior at $T=0$ from half-integer-spin chains. Specifically, Haldane expects integer-spin chains to show a gap in their excitation spectrum in the thermodynamic limit whereas the spectrum for half-integer-spin Heisenberg chains is gapless, as for the exactly solvable case of spin-1/2.

Heisenberg Hamiltonians with $S>1 / 2$ are apparently nonintegrable, unlike the special case of spin-1/2. A number of finite-chain numerical treatments have appeared ${ }^{2-4}$ which show features consistent with the Haldane picture. However, the evidence is suggestive rather than conclusive, and essentially the same numerical evidence is open to interpretation in quite different ways. ${ }^{2,3,4}$ The Haldane conjecture has dramatic implications since it is in conflict with traditional concepts such as the validity of Kubo-Anderson spinwave theory.

It also implies that proceeding from the quantum to the classical limit is nontrivial, a major feature of interest here. ${ }^{5}$ The question of quantum-classical crossover has arisen in a different context. Recent work on the low-temperature dynamics of the spin-1/2 Heisenberg antiferromagnetic chain has revealed a wealth of novel phenomena for this quantum system, including spin-wave double continua, of importance to the structure factor, and the presence of soft modes which "track" across the Brillouin zone as the applied field increases from zero to saturation. ${ }^{6}$ Such features are not observed in classical Heisenberg spin chains. ${ }^{6}$ Our purpose has therefore been to mount the most comprehensive investigation extant of the spectral excitations and static properties,

\footnotetext{
a) On leave from Department of Mathematics, UMIST, P.O. Box 88, Manchester M60 1QD, UK.

b) On leave from Physics Department, University of Rhode Island, Kingston, RI 02881 .
}

including the $T=0$ magnetization curves versus field and the real-space correlation functions $\left\langle S_{i}^{z} S_{i+R}^{z}\right\rangle$ and their Fourier transforms (essentially the integrated intensities) of Heisenberg chains of spin-1/2 and higher spin. The exact finite chain calculations have been sufficient to elucidate an interesting and unanticipated crossover mechanism with increasing spin from quantum to classical behavior. In the zero field case, however, hints of behavior consistent with Haldane's prediction are observable in the longest chains for a given spin value. Hence investigations have proceeded on spin-1 chains of much larger $N$ using a new Monte Carlo method.

Exact finite chain calculations have relied principally on a Lanczös technique ${ }^{7}$ to find the lowest eigenvalues for each allowed value of the wave vector $q$ and also each value of the $z$ component of the total spin $S_{T}^{z}=\Sigma_{i} S_{i}^{z}$ which essentially yields the complete $T=0$ field-dependent behavior. Eigenvectors have also been calculated. For the case of spin$1 / 2$ we have data through $N=20$ for the eigenvalues and through $N=14$ for the correlations. ${ }^{8}$ For spin- 1 data exists through 12 spins, for spin- $3 / 2$ through $N=10$, and for spin2 through $N=8$ for both eigenvalues and correlations, and for spin-1, $N=14$, eigenvalues only. Monte Carlo data exist (at present) for spin-1, $N=12,16,24$, and 32, for spectral excitation gaps only.

\section{QUANTUM-CLASSICAL CROSSOVER}

In Fig. 1 we show the lowest-lying energy states relative to the ground state for spin- 1 and $N=14$, as a function of $q$ for a sequence of $N S-1, \ldots, N S-N / 2=7$ values of $S_{T}^{z}$ (essentially the $T=0$ magnetization per spin). The feature to observe is the "quasi-soft" mode which tracks across the Brillouin zone from $q=0$ to $q=\pi$ as $S_{T}^{z}$ decreases. (It can be shown by extrapolation that the quasi-soft modes have 


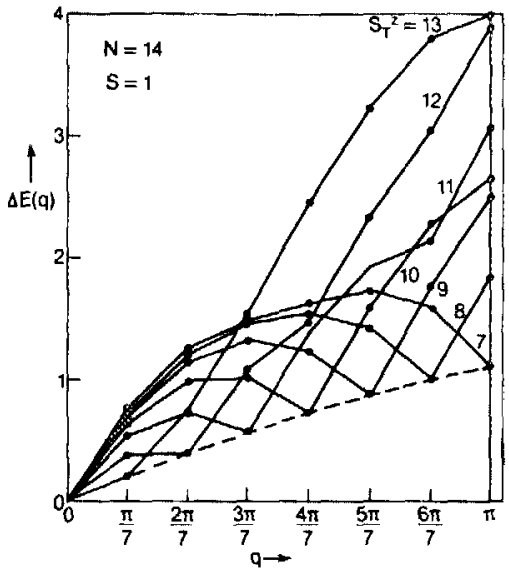

FIG. 1. Lowest-lying excitations for $S=1, N=14$ vs $q$ for the "quantum regime," $14>S_{T}^{z}>7$. The dominant "soft modes" lie on a smooth curve (shown dashed) which extrapolates to zero as $N^{-1}$.

zero energy gap in the thermodynamic limit.) Figure 1 is qualitatively similar in every feature to the corresponding plot for spin-1/2, $N=14$, except that, in the case of spin-1/ 2 , all $S_{T}^{2}$ values and hence all the lowest-lying states are exhausted by such a plot. Since the plot for spin- $1 / 2$ is inherently quantum we deduce that a fraction $(1 / 2)$ of the dispersion curves for spin-1 also possess quantum character. In fact, we find this is a general result for all spin, i.e., a fraction $1 / 2 S$ of the dispersion curves close to the saturation magnetization value (or, equivalently, the antiferromagnetic saturation field) have this quantum character. An appreciation of the behavior of the nonquantum states (for $S>1 / 2$ ) is gained from Fig. 2 which is a companion plot to Fig. 1. A striking qualitative semiclassical character is apparent in the dispersion curves, where a gap is present at the zone boundary which increases with increasing $S_{r}^{z}$ (or, equivalently, field). These observations, which are consistent with behavior observed also for spin values $3 / 2$ and 2 , indicate that quantum effects exist for all $S<\infty$, vanishing as $(2 S)^{-1}$ in the classical limit. Support comes from the $T=0$ magnetization curves of Fig. 3, which show approximations to the $N \rightarrow \infty$ magnetization curves for $S=1$ and $S=1 / 2$ obtained by

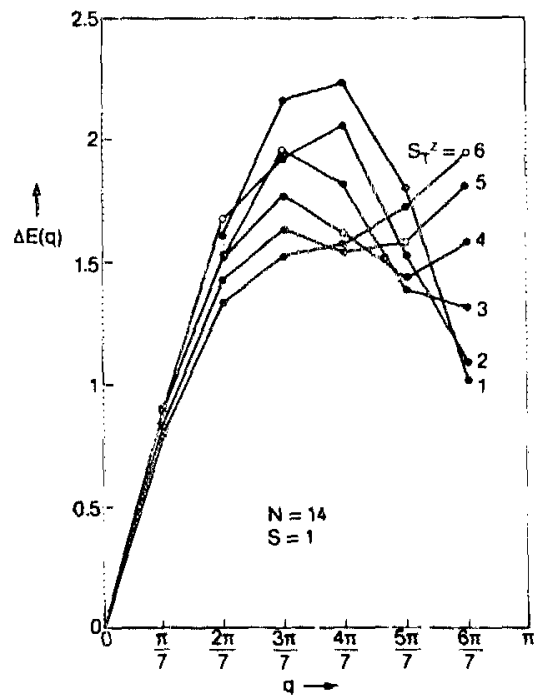

FIG. 2. Lowest-lying excitations for $S=1, N=14$ vs $q$ for the "classical regime," $7>S_{T}^{z}>0$.

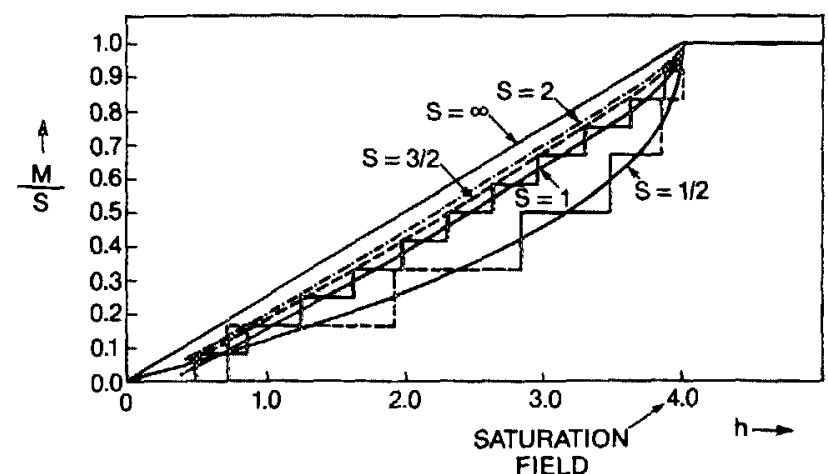

FIG. 3. The singlet-triplet (primary) excitation gap for various spin chains vs $N^{-1}$. The points with error bars are preliminary Monte Carlo results for $S=1$.

drawing smooth curves through the step midpoints for the corresponding $N=12$ step functions. The pronounced quadratic character of the $S=1 / 2$ curve over about half of the range near to saturation is seen also in the companion spin-1 curve but over a smaller part of the range, consistent with a quantum regime extending from $M_{\text {sat }}$ to $M_{\text {sat }}(1-1 / 2 S)$. The remainder of the spin-1 curve is remarkably linear, a classical characteristic as demonstrated by the $S=\infty$ curve shown also in Fig. 3. Smooth midpoint curves for $S=3 / 2$ and $S=2$ confirm the effect. Finally, we comment that this quantum-classical effect appears also in the Fourier transform of the correlation functions, which is essentially the experimentally observable integrated intensity. ${ }^{5}$

\section{THE HALDANE CONJECTURE}

In Fig. 4 we show the exact values for the primary singlet-triplet $(0,1)$ excitation exergy gap as a function of $1 / N$ for various spin chains. These results check earlier calculations ${ }^{2}$ and extend them in the case of spin $1 / 2$ by including points up to $N=20$, and $N=14$ for $S=1$. For the case of $S=1 / 2$, the finite $N$ points show concave downwards curvature with a trend towards zero (no gap). The points for $S=1$, on the other hand, show opposite, concave upwards, curvature. If

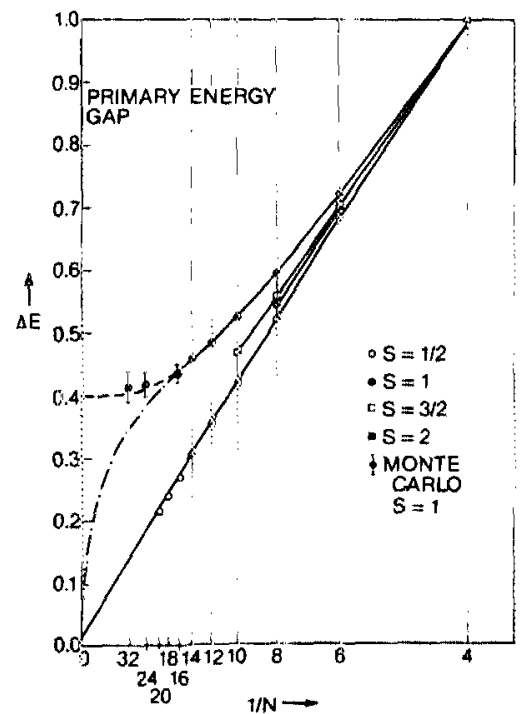

FIG. 4. $T=0$ magnetization curves vs reduced field $h=g \mu_{B} H / J S$ estimated for $S=1 / 2,1,3 / 2,2$, compared with $S=\infty$ (exact). The behavior near $h=0$ relates directly to the Haldane conjecture. Available results are not, at present, conclusive and therefore are not presented on the figure. 
this trend continues into the asymptotic large- $N$ region, the existence of a gap is definitely indicated. The case of $S=3 / 2$ is interesting in that a smooth curve through the available points for $N=4$ through 10 would show an inflection point. For small $N$ the curvature is similar to the case of $S=1$, and only for large $N$ does a downward turn set in indicating a crossover in trend towards gapless behavior. Not enough points are available for $S=2$ to draw conclusions. The above features are definitely consistent with Haldane's prediction. ${ }^{1}$ However, caution is advised in that since the points for $S=3 / 2$ show an "inflection" point, the points for $S=1$ may eventually do so also, at a relatively higher value of $N$. Hence the calculation of such higher $N$ points is definitely indicated.

Much existing numerical work has focussed on the "primary" singlet-triplet energy gap. ${ }^{2,3}$ For the farniliar exactly solvable, i.e., exactly integrable, models, higher excited gaps will bear a simple numerical relationship to the primary gap. This is not necessarily the case for nonintegrable systems, however, ${ }^{9}$ and hence we have investigated gaps corresponding to higher excitations above the singlet ground state, specifically the minimum singlet-quintet $(0,2)$, singletseptet $(0,3)$ and singlet-singlet $(0,0)$ gaps for spin- 1 . None of these "higher gaps" shows a trend to zero, behaving roughly as the primary gap although with somewhat different convergence properties.

The unknown territory of nonintegrable systems suggests that a prudent course is not to rely on a single feature, such as the (primary) gap but to comprehensively survey all relevant properties of the system. The zero-field integrated intensity $S(q)$ is the space Fourier transform of the zero-field pair correlations $\left\langle S_{i}^{z} S_{i+R}^{z}\right\rangle$. For spin-1/2, the integrated intensity is expected to diverge at $T=0$ at the zone boundary,

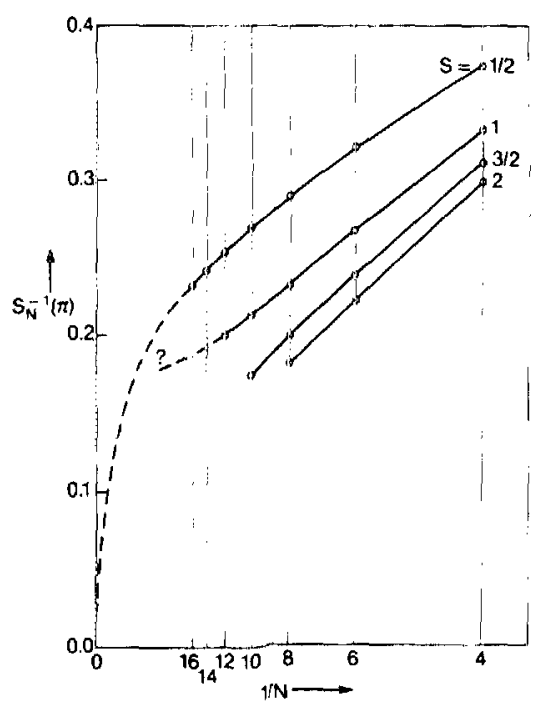

FIG. 5. Plots of $S_{N}^{-1}(\pi)$ vs $N^{-1}$ for various spin values, as indicated. $q=\pi$, as $\ln N$ with additional $\log$ corrections. For a system with a gap, $S_{N}(\pi)$ will remain finite in the limit $N \rightarrow \infty$. For comparative purposes with the energy gap extrapolations we have plotted $\left[S_{N}(\pi)\right]^{-1}$ vs $1 / N$ in Fig. 5 for various spin chains. For spin-1/2, the trend of the points is smooth, increasing curvature with increasing $N$ indicating a limiting zero value ${ }^{10}$ (trend shown dashed). For spin-1, on the other hand, the presence of an "inflection" point is a definite indication that the limiting extrapolated value is finite. The available points for spin- $3 / 2$ reflect those of spin-1/2, indicating trend to zero and, again, the points for $S=2$ are too few to be informative. Hence, once more, the available evidence is consistent with the Haldane picture, although longer chain information is desirable. The special significance here is that $S(\pi)$ depends only on ground state properties since it is calculated from the ground state eigenfunctions.

Finally, we applied a Monte Carlo technique, which yields eigenvalues for quantum spin chains. ${ }^{11}$ Also in Fig. 1, the resulting estimates and error bars are shown for the spin-1 primary gaps for $N=16,24$, and 32 . These preliminary results lack sufficient accuracy to verify Haldane's conjecture or falsify it unambiguously: the data are consistent with an exponential trend in $N$ to a nonzero gap (dashed curve in Fig. 1), but an inflection point followed by an ultimate trend to zero (dot-dashed curve) is not completely ruled out. The results are promising and additional calculations are in progress.

We acknowledge support from the NSF Visiting Professorships Program (JCB), NSF grants DMR80-10819, DMR84-06186, the ONR, and NATO.

'F. D. M. Haldane, Bull. Am. Phys. Soc. 27, 181 (1982); Phys. Rev. Lett. 50, 1153 (1983); Phys. Lett. A 93, 464 (1983).

${ }^{2}$ R. Botet and R. Jullien, Phys. Rev. B 27, 613 (1983); M. Kolb, R. Botet, and R. Jullien, J. Phys. A 16, L673 (1983); M. Kolb, R. Botet, and R. Jullien, Phys. Rev. B 29, 5222 (1983).

${ }^{3}$ U. Glaus and T. Schneider, Phys. Rev. B 30, 215 (1984).

${ }^{4}$ J. C. Bonner and G. Müller, Phys. Rev. B 29, 5216(1984); S.-T. Chui and K. B. Ma, Phys. Rev. B 29, 1287 (1984); J. Solyom and T. A. L. Ziman, Phys. Rev. B 30, 3980 (1984).

SJ. C. Bonner and J. B. Parkinson (unpublished work). A preliminary account has appeared in the Proceedings of the Workshop on Elementary Excitations and Fluctuations in Magnetic Systems, San Miniato, Italy, 1984 (Springer, New York, 1984), p. 55.

${ }^{6} \mathrm{G}$. Müller, H. Thomas, H. Beck, and J. C. Bonner, Phys, Rev. B 24, 1429 (1981).

${ }^{7}$ T. Sebe and J. Nachamkin, Ann. Phys. 51, 100 (1969).

${ }^{8}$ For spin-1/2 only, Lanczös techniques can be supplemented by Bethe Ansatz calculations for longer chains.

${ }^{9}$ B. M. McCoy (unpublished work). One of us (G.M.) is indebted to B. M. McCoy for alerting him to the implications of nonintegrability in statistical models.

${ }^{10}$ Data for $S=1 / 2$ and $N=16$ were obtained from W. Grieger, Phys. Rev. B 30, 344 (1984) and J. Borysowicz, T. A. Kaplan, and P. Horsch (unpublished work).

${ }^{11} H$. W. J. Blöte and M. P. Nightingale (unpublished work). 